\title{
Globe
}

Revue internationale d'études québécoises

\section{Cinq livres. Un débat}

\section{Michel Despland}

Volume 11, numéro 1, 2008

URI : https://id.erudit.org/iderudit/1000501ar

DOI : https://doi.org/10.7202/1000501ar

Aller au sommaire du numéro

\section{Éditeur(s)}

Globe, Revue internationale d'études québécoises

\section{ISSN}

1481-5869 (imprimé)

1923-8231 (numérique)

Découvrir la revue

Citer cette note

Despland, M. (2008). Cinq livres. Un débat. Globe, 11(1), 237-253.

https://doi.org/10.7202/1000501ar d'utilisation que vous pouvez consulter en ligne.

https://apropos.erudit.org/fr/usagers/politique-dutilisation/ 


\section{NOTES CRITIQUES}

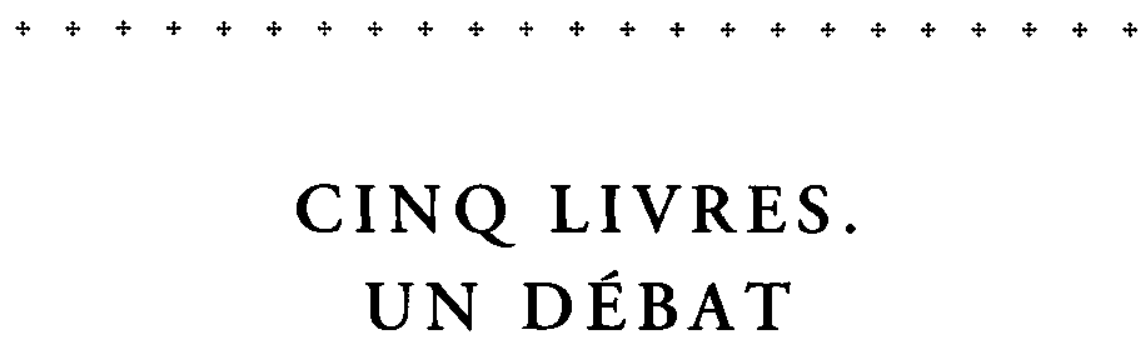

\section{MICHEL DESPLAND \\ Université Concordia}

$4+4+4+4+4+4+4+4+4+4+4+4$

L'Église catholique, écrit Georges Balandier, «est la plus puissante des machines sociales opposées au temps ${ }^{1}$ ». Le Québec semble offrir une bonne illustration de cette généralisation, du moins à en croire les clichés qui circulent sur la Révolution tranquille qui a commencé en 1960 avec l'élection du gouvernement de Jean Lesage. Qui n'a pas entendu parler de la "grande noirceur", de la "chape de plomb" que l'Église faisait peser sur les Québécois? E.-Martin Meunier et Jean-Philippe Warren² s'élèvent contre cette simplification outrancière, qui est un mythe plus qu'un outil d'analyse. La démonstration a trois parties. Bien avant 1960, un grand glissement s'est fait chez les penseurs catholiques, passant de l'éthique du concile de Trente à l'éthique personnaliste. Le personnalisme a son origine chez les catholiques français soucieux de surmonter l'opposition entre un traditionalisme de droite chantant la cohésion de la nation et un individualisme délétère et égoïste. Le personnalisme défendait à la fois la conscience individuelle et la solidarité sociale. Le mouvement invitait donc à l'action sociale en faveur de

$$
+4
$$

1. Georges BalandieR, Le dedale. Pour en finir avec $k$ Xx sizcke, Paris, Fayard, 1994, p. 161.

2. E.-Martin MEUNIER et Jean-Philippe WARREN, Sortir de la *Grande Noirceurs. Lhonzon "personnalsse* de la Révolution tranquille, Sillery, Septentrion, 2002. 
la justice pour tous. Ainsi, à une éthique insistant sur le poids du péché et la médiation de l'Église en succédait une autre qui appelait à l'action pour mieux habiter le temps et y accomplir tout le potentiel humain. La diffusion au Québec de ces idéaux dans l'intelligentsia catholique se démontre sans peine; ainsi naquit ici l'utopie d'une nouvelle chrétienté. En un second temps, les auteurs soulignent avec Max Weber la force mobilisatrice de la religion. La nouvelle éthique invite à une ascèse personnelle débouchant sur une action sur le monde à la fois consciencieuse, compétente et efficace. C'est tout le Berufallemand, c'est-à-dire la vocation pour une activité professionnelle et intramondaine, qui entre dans le monde de la religion catholique. Ceux d'entre nous qui ont connu les nouveaux serviteurs de l'État à l'œuvre dans la foulée de la Révolution tranquille seront d'accord avec cette affirmation. Ce que l'on voit alors au Québec, c'est non seulement une offre religieuse venant d'en haut, mais aussi une demande religieuse présente en bas. En un troisième temps, on nous montre non comment la société québécoise devait changer, mais comment l'Église l'a voulu. Les débats sur la création du ministère de l'Éducation (1964) le montrent bien; on pourrait aussi l'illustrer avec l'histoire de la sortie de l'Église de la gestion des hôpitaux.

Meunier et Warren écrivent donc un meilleur roman des origines de la modernité au Québec. Ils y retrouvent une sortie religieuse de la religion qui n'est pas sans rappeler l'argument de Marcel Gauchet, autre grand lecteur de Max Weber. Ils enrichissent la lecture sociologique avec un examen de la motivation des personnes et l'histoire qu'ils présentent inclut des agents aussi bien que des forces sociales. De plus, on y rencontre des sujets qui luttent pour accéder à l'universel et travaillent pour traduire un idéal dans les conditions de leur lieu et de leur temps. Mais en ouvrant ces pages parfois exaltantes, les auteurs finissent par nous amener à des constatations maussades. La Révolution tranquille eut des effets non voulus qui vite apparurent indésirables aux plus idéalistes des acteurs. La modernisation apporta la bureaucratie ainsi que le nouveau monde de la consommation niaise et de la culture de masse. Les Québécois boivent moins de bière et de gros gin, mais plus de grands crus français; était-ce là le but des luttes? Les personnalistes connurent donc le désenchantement après l'action. L'abbé Grand'Maison écrivit en 1978: "Nous faudra-t-il aller dans le Tiersmonde [sic] pour redécouvrir l'homme nu, vrai et passionnant? Le nonhomme n'est pas là-bas, mais chez nous. Oui chez nous, l'homme le plus nié, 
le plus vidé, le plus aliéné ${ }^{3}$ " Les essais de Jean Larose publiés en 1987 sous le titre La petite noirceur montrent que noirceur et modernité ne s'excluent nullement; on peut être à la fois bien nanti et moralement et politiquement faible ${ }^{4}$. L'acquis de cet essai me semble être l'affirmation que ce que l'on appelle la religion est, dans les faits, une réalité ambiguë dont les effets sont équivoques. Un essai tout à fait apte à relancer les débats dans la société québécoise, dont Fernand Dumont disait en 1990 qu'elle était "en panne d'interprétations".

Raymond Lemieux et Jean-Paul Montminy ${ }^{6}$ sont deux sociologues chevronnés qui veulent faire le point : le catholicisme peut-il être porteur de projets pour les Québécois aujourd'hui? L'année 1960 marquet-elle le début d'une disparition ou le commencement d'une résurgence? La démarche se déroule en trois étapes. Tout d'abord, un chapitre historique veut rééquilibrer l'histoire de l'Église catholique au cours du XIX ${ }^{e}$ siècle. L'Église fournit une matrice culturelle au Québécois après l'échec politique et militaire de 1837-1838. Cette matrice encouragea une vision identitaire dualiste: il y a nous et il y a les autres, c'est-à-dire au premier chef les angloprotestants. La dynamique identitaire se déploie en fonction de dangers: les Anglais, l'industrialisation, l'urbanisation. Les dangers ne sont pas tous inventés: le rapport Durham indique les moyens d'assurer la disparition de l'identité canadienne-française ("peuple sans histoire et sans culture") grâce à une assimilation douce et progressive ${ }^{7}$. Le deuxième volet documente la situation depuis 1960: il existe une crise de crédibilité pour l'Église, attestée par la chute de la pratique et le déclin des vocations à la vie sacerdotale ou religieuse. L'Église cherche dès lors à se recentrer sur sa mission propre et à s'investir dans le "marché du sens". Vient le troisième volet. Selon les bonnes méthodes de l'Action catholique, les auteurs, après avoir vu et jugé, agissent. Ils définissent les défis. On ne sait plus qui est dans et qui est hors de la communauté catholique. Très rares depuis 1965 sont les catholiques qui acceptent la vision pontificale de l'éthique sexuelle. Ils ne se sentent pas moins

$$
+4
$$

3. Jacques Grand'MAISON, Quelle société?. Montréal, Leméac, 1978, p. 33; reproduit sur le site suivant: hitp:/I classiques.uqac.ca/concemporains/grandmaison_jacques/quelle_societe/quelle_sociere.doc\#quelle_societe_chap_1_2e (15 octobre 2007).

4. Jean LAROSE, La petite nourceur, Montréal, Boréal, 1987.

5. Fernand DUMONT [dir.], La société québécose après 30 ans de changements, Québec, Institur québécois de recherche sur la culcure, 1990, p. 14. Sur Fernand Dumont, voir Jean-Philippe WARREN, Un supplement d'àme Les intentions primordiales de Fernand Dumont (1947-1970), Québec, Presses de I'Université Laval, 1998.

6. Raymond LemIEUX et Jean-Paul MONTMINY, Le casholictsme quebecois, Quebec, Éditions de l'IQRC, 2000.

7. Gérard Bouchard parle néanmoins de la construction d'une fausse idencité (La nation québécosse au futur et au passé, Montréal, VLB éditeur, 1999, p. 130). 
catholiques pour autant. La visite du pape en 1984 déplace d'énormes foules, mais ses enseignements socioéconomiques trouvent très peu d'échos. L'examen des croyances montre que beaucoup de catholiques acceptent la réincarnation et autres conceptions dénoncées par les Pères de l'Église depuis le troisième siècle. Une identité demeure indubitablement: on se dit catholique, mais le sens de la déviance n'existe pas. Le problème n'est donc pas de croire, mais de croire ensemble. Le témoignage d'un curé est éloquent: les gens viennent chercher le baptême pour leurs enfants comme si c'était un droit. (Comme pour le passeport canadien, sauf que c'est un autre guichet.) Certes, la foi continue à vivre, mais hors des niches traditionnelles. Les directions offertes par le rapport Dumont (1971) restent valables: une radicalité des options de vie doit continuer à s'affirmer. Il y a les engagements caritatifs auprès du quart-monde, l'œcuménisme pragmatique, de terrain. Mais, surtout, il y a la défense du droit pour tout humain de penser et de donner un sens à sa vie et l'invitation à travailler à l'exercice de ce droit dans un appui mutuel. Il reste encore le choix "entre l'absurde et le mystère".

Les auteurs s'avancent courageusement sur un terrain miné en affirmant (dans le titre de leur seconde partie) que le catholicisme est la "culture primordiale" des Québécois. L'adjectif étonne. On connaît la formule de George Orwell dans La ferme des animaux: "Tous les animaux sont égaux, mais certains sont plus égaux que d'autres ${ }^{8}$." La Charte des droits et libertés affirme sans équivoque que tous sont égaux. Par ailleurs, dans les faits, les catholiques (nominaux pour beaucoup, on vient de le voir) sont la grande majorité de la population. En France, nul ne s'offusque d'entendre dire que la France est la fille aînée de l'Église, car on sait bien que les filles s'émancipent. Mais comment traduire la donnée quantitative de la démographie dans du qualificatif proprement québécois? Les carholiques sont-ils les porteurs et gardiens d'une identité nationale à laquelle les nouveaux venus doivent s'adapter? Le problème est clairement posé depuis l'essai de Gérard Bouchard intitulé La nation québécoise au futur et au passé, qui oppose la conception ethnique de la nation et la conception civique. Si la première est devenue inacceptable, la seconde est un peu courte'. On n'a jamais su dire

$$
+\div
$$

8. George ORWELL, La ferme des anmaux, Paris, Gallimard, 1983 [1945], p. 144.

9. Par delà la "domınation" exercée par le clergé, Louis Rousseau a su montrer l'élaboratıon entre 1840 et 1880 d'une ethnicıté et d'une identité à forte composante religieuse chez les Canadiens français. Voir Louss ROUSSEAU, «La place du religieux dans la naissance et le déplacement de l'image identıtaire au Québec, 1840-1980n, Claude SORBETS et Jean Pierre Augustin [dir.], Préférences politagues et références culturelles au Canada, Sainte-Foy/Bordeaux, Presses de l'Unıversité Laval/Massons des Sciences Humaines d'Aquitaine, 2001, p. 49-76; "La construction religieuse de la narion", Recherches sociographiques, vol. XLVI, $n^{\circ} 3,2006$, p. 437-452. Il parle même d'une "genèse de la société québécoise en tant que Nation-Eglıse " dans « Périodiser l'histoire religieuse du Québec: Questions épıstémologiques et 
exactement comment on devient Canadien français sinon par la naissance: parler la langue ne suffit pas. Qu'est-ce qu'il y a dans la convivialité ethnique des Canadiens français qui peut déteindre sur les autres? Lemieux et Montminy donnent des éléments de réponse en soulignant qu'après avoir enseigné à tous la résignation et la résistance, l'Église a enseigné (à une élite) un esprit activiste de conquête dans ce monde. Il est aussi vrai que les encycliques et déclarations de Jean XXIII et de Paul VI sur la nature et les tâches de l'État affirment des valeurs universelles et poussent à l'action citoyenne. Il n'en reste pas moins, concluent-ils, que la lä̌cité au Québec (de l'an 2000) reste à construire ${ }^{10}$. Ajoutons qu'à cette date, le pluralisme religieux n'apparaît à ces auteurs que sous le visage des groupuscules périphériques qui séduisent quelques catholiques. Les choses ont bien changé depuis.

Les deux livres déjà présentés sont des essais de 207 et 141 pages. J'en propose maintenant deux autres qui ont, respectivement, 860 et $504 \mathrm{pa}$ ges. Le genre littéraire change; on passe à de gros travaux universitaires.

Gilles Havard ${ }^{11}$, un diplômé de l'Université Laval qui enseigne à Paris, nous donne Empire et métissage. Indiens et Français dans le Pays d'en Haut 1660-1715. Je souhaite attirer l'attention sur les thèmes de la troisième partie et passerai rapidement sur les deux premières qui ne nous intéressent qu'indirectement. Examinant la nouvelle géopolitique régionale établie au cours du XVII siècle dans le pays autour des Grands Lacs (qui touche aux plaines de l'Ouest et descend jusqu'au golfe du Mexique), l'auteur nous montre comment les Français rencontrent un système politique et économique complexe de plusieurs "nations" qui oscillent entre guerres et alliances. Ce qu'il faut retenir ici c'est que les acteurs allochtones (agents royaux, commerçants et missionnaires) rencontrent un monde autochtone diversifié. Face à cet univers multipolaire, la dynamique d'un rapport entre nous et les autres (celle qui sera renforcée au XIXe siècle par les dogmes racistes, biologiques et darwiniens) ne saurait s'installer. Les Français s'insinuent dans un système d'alliances et construisent leur pouvoir sur l'attrait des marchandises qu'ils apportent et l'offre de médiations visant à

$$
+\div+
$$

propositionsn, Anne ForTIN et François Nault [dir.], Dire limpensable, l'Autre. Ptrégrinations avec Raymond Lemteusx, Montréal, Médiaspaul, 2004, p. 273-305.

10. Jacques Grand'Maison avançait en 1979 que le débat sur la place de la religion n'a tour sımplement pas eu lieu au Québec er que la société se condamnạit ainsı à entrer dans l'avenit à reculons. ll ajoutait que le mouvement lasque s'est sabordé, croyant, non sans rasson, que la sécularısation allait se faire toute seule. (Jacques GRAND'MAISON, «Un sous-sol historique mal assumén, Une foi ensouchée en ce pays, Montréal, Leméac, 1979, p. 137-139.)

11. Gilles Havaro, Empire et métussages. Indiens et Français dans le Pays d'en Haut 1660-1715, Sullery, Septentrion, 2003. 
agrandir «le cercle de la paix». La deuxième partie, «Une marge impériale en pays indien", montre comment l'empire vit dans la contradiction. Le discours royal est impressionnant et la volonté de conquête patente. Louis XIV est censé être un grand roi invincible sous la protection duquel tous ses peuples prospèrent. Les autochtones entrent dans le jeu de la protection, mais pour leurs propres fins. Dans les faits, le système est très faible: les ordres de Versailles n'arrivent que lentement à Québec et ceux de Québec mettent longtemps pour arriver à Michillimakinac; de plus ce que l'on appellerait de la corruption est omniprésent. Il n'y a pas vraiment d'appropriation française du territoire; les postes sont des ambassades et non des centres administratifs. Les Français réussissent à y "mettre la force en signes", mais le réseau d'obligations ainsi construit reste faible.

La table est ainsi mise pour la troisième partie, "Échanges et métissages dans l'empire du milieu ". Les Canadiens français, rappelons-le, sont le seul peuple d'Amérique du Nord qui donna naissance (au sens littéral du mot) à une nation métisse, qui fut écrasée militairement en 1885, alors que le gouvernement canadien entendait peupler les Grandes Plaines d'une autre manière. Havard nous montre le pourquoi et le comment des pratiques de métissage dans ces vastes territoires. Échanges commerciaux et diplomatie des cadeaux tissent et maintiennent un réseau social, et même des attachements, qui ignorent les fictions de l'empire. L'écart culturel est vaste. Les Français ont un État, alors que les Amérindiens n'ont pas d'institution autorisée à imposer une coercition. Les premiers croient que la maladie est une pénitence, les seconds pensent qu'elle résulte du non-assouvissement de désirs. Mais les conditions de vie pratique forcent les Français (missionnaires y compris) à s'habiller, manger, voyager et se loger comme les Indiens. Dans des situations de détresse ou de disette, les allochtones abandonnent le masque d'agents de l'empire, font appel à la pitié des autochtones et obtiennent du secours. Bref, dans un nouvel espace, au milieu, ils "s'ensauvagent ", pour utiliser le terme que l'élite de la vallée du Saint-Laurent utilise dans les rapports envoyés en France. L'histoire des pratiques sexuelles est la manifestation la plus forte de l'hybridation qui s'invente chaque jour dans les conditions d'innovation (Havard parle même d'effervescence sociale et culturelle) propres au Pays d'en Haut. On sait qu'il y avait pénurie de femmes françaises (des estimations donnent en 1663 une femme pour six ou sept hommes). Du côté amérindien, la guerre causait un déséquilibre dans l'autre sens, moindre évidemment, et les jeunes femmes avaient la libre disposition de leur corps. L'inévitable se produisit: l'éventail des pratiques allait de l'occasionnel jusqu'au mariage catholique. Les coureurs des bois 
négociaient des cohabitations à terme. Les femmes connaissaient le terrain, servaient d'interprètes; elles préparaient la nourriture de leur homme et aidaient à porter les canots. L'utile assaisonnait donc l'agréable (à moins que ce ne soit l'inverse). Les mariages étaient encouragés de part et d'autre. Le gouverneur Vaudreuil fait exception en déplorant l'affaiblissement du sang. Ainsi s'opposaient le métissage et l'alliance à l'empire, avec ses rêves d'homogénéité. Les conditions matérielles de l'existence et le poids de la solitude, bref la vie, faisaient sauter les cadres de la société et les exigences de l'idéologie. Les jésuites s'engagèrent dans une acculturation raisonnée. Ils devinrent experts avec les pagaies, mais ne frayaient pas avec les sauvagesses.

La religion, logée dans l'absolu, est pour les Français ce qui échappe entièrement aux logiques de l'échange et du don. Et pourtant, les allochtones adoptent la pratique du tatouage et l'un d'entre eux arbore une Vierge à l'enfant et une croix sur l'estomac ainsi qu'un serpent qui fait le tour du corps. Au passage dans des sites sacrés, alors que les autochtones font une offrande de tabac, les nouveaux venus font aussi un geste rituel, mais avec de l'eau. Havard montre comment les Français retrouvent dans le Pays d'en Haut les pratiques magiques de leurs campagnes natales et se mettent à faire des offrandes de tabac. L'auteur reste assez bref à ce chapitre, mais il ouvre la possibilité d'enquêtes méticuleuses sur la vaste série des phénomènes de métissage. Ainsi, il devient pertinent d'explorer l'apport des Irlandais au catholicisme québécois et de savoir qu'il y avait des mariages mixtes catholiques-protestants après la Conquête et avant 1837. Jacques Bouveresse écrit qu'une logique pratique, celle du flou et de l'à-peu-près, définit le rapport ordinaire au monde ${ }^{12}$. (Les idéologies apportent ensuite leurs clartés souvent inquiétantes.) Havard nous montre, peut-être involontairement, que "la religion" connaît aussi le flou. Une page de Lionel Groulx oppose le monde de la terre, qui stabilise et enracine, et le monde de l'eau, qui ensorcelle et disperse; un monde où tout bouge ${ }^{13}$. Harvard nous fait voir ce qui bougeait dans les profondeurs de la psyché.

Empire et métissage élargit l'interprétation de la religion au Canada français en ouvrant l'enquête au niveau de microphénomènes sociaux. Alors que la sociologie de Durkheim voit la vie sociale surtout sous l'angle de la

$$
\div \div
$$

12. Jacques BOUVERESSE, Bourdieu, savant et politique, Marseille, Agone, 2003, p. 161.

13. Jean-François Mouhor trouve crois phases dans la perception de l'influence amérindienne par les historiens canadiens: années maigres, années de la négarion et annés fastes. Lionel Groulx appartient à la seconde. (Jean-François MouHOT, "L'influence amérindienne sur la société en Nouvelle-France. Une exploration de l'historıographie de François-Xavier Garneau à Allen Greer (1845-1997) n, Globe. Revue internationale d'trudes québtcosses, vol. 5, n 1, 2002, p. 123-157.) 
contrainte, celle de Havard inclut la socialité libre et quotidienne, celle de l'interaction entre individus qui se rencontrent. Cette dernière s'improvise au fil des circonstances et gère les matérialités et les aléas ${ }^{14}$. Depuis une dizaine d'années, des auteurs ont recours à l'idée de mérissage pour rendre compte de la fluidité de ce qui se passe dans des quartiers montréalais ${ }^{15}$.

L'ouvrage collectif assemblé par Jean-Marc Larouche et Guy Ménard, L'étude de la religion au Québec ${ }^{16}$, fait état d'un bouleversement dans l'interprétation de la religion en montrant un changement de paradigme. Une révolution copernicienne a entrepris de transformer la base même de la rationalité (ou de la scientificité) du discours sur la religion. Au lieu de mettre en valeur l'universel visé par la religion catholique, il s'agit dès lors de dresser un atlas "universel " de ce que l'on trouve empiriquement dans le temps et sur la surface de la terre. En d'autres termes, il s'agit de recenser l'ensemble des religions dites "du monde" et dont plusieurs attestent aujourd'hui la présence à l'intérieur des frontières du Québec, terre d'accueil. Après de modestes débuts au cours des années 1970, une génération de chercheurs ont voyagé, appris les langues, acquis et affiné de nouveaux outils de recherche, si bien qu'un grand nombre de nouveaux enseignements sont entrés dans le quotidien des universités ${ }^{17}$. Parler de «sciences humaines de la religion» marqua la nouveauté.

Le grand recueil de Larouche et Ménard (trente-cinq chapitres) révèle l'ampleur des travaux accomplis (et publiés) ainsi que la variété des connaissances disponibles ${ }^{18}$. Une première section porte sur les traditions religieuses de l'humanité. Douze y sont présentes. Une deuxième aborde les textes sacrés et les traditions religieuses anciennes; plus traditionnellement philologique, ce groupe de chercheurs poursuit les travaux en études bibliques et patristiques déjà présents dans les facultés de théologie depuis un

$$
+4+
$$

14. Ce type de regard sur le social remonte au grand essai de Marcel Mauss, "Essai sur le don \# (1925), dans Sociologze et anthropologue, Paris, Presses universitaures de France, 1950. Sur Mauss, voir Camille TAROT, Socsologze et anthropologie de Marcel Mauss, Paris, La Découverte, 2003. René Callé er lacques Godbouc onc fait fructifier cet héricage dans Critrque de la ratson utstatre (Paris, La Découverte, 1989) er dans Le don, la dette et l'sdentité Homo donator us bomo aconomicus (Montréal/Paris, Boréal/La Découverte, 2000).

15. Pour une illustration de la montée du transculturel dans la vie des arts, voir les numéros de la revue Vice Versa publiés de 1983 à 1996 et Sherry SIMON, Hybridité culturelle, Montréal, lie de la Tortue, 1999.

16. Jean-Marc LAROUCHE et Guy MÉNARD [dir.], L'étude de la relugton au Québec. Bulan et prospective, Québec, Presses de l'Université Laval, 2001.

17. On trouve les premiers foyers de développement à l'Université du Québec à Moncréal (UQAM), avec le programme de relıgıologie, et à l'Université Laval, avec la sociologie religieuse. Ces deux universités ont lancé la revue Religuologtques et les Cahters de recherche en sciences de La religion. Aujourd'hui, Concordia, I'UQAM ex Laval offrent un programme conjoint en sciences des relıgions.

18. Une comparason peur être faire avec le recueil Religion et culture au Quebec Figures contemporaines du sacré, sous la direction d'Yvon Desrosiers (Montréal, Fides, 1986). 
demi-siècle. Viennent ensuite deux parties plus thématiques: «Les nouvelles manifestations du religieux dans la culture" et "Religion, pratiques sociales et productions culturelles". Cette dernière s'achève avec un article sur la rencontre des cultures et des religions.

L'introduction magistrale de Louis Rousseau commence par une note de fierté. Ce bilan atteste la présence d'un domaine d'études dont l'élan fut depuis quarante ans l'œuvre de plusieurs vies qui ont formé la relève. Ce domaine a conscience de soi, produit des outils collectifs et rayonne à l'étranger. Rousseau souligne, avec raison, que ce qui se passe dans ce canton du travail savant, c'est une redéfinition de la fonction sociale de la religion ainsi que du travail de l'intelligence en ce domaine. Mais il ajoute une note d'inquiétude: les gestionnaires universitaires vont-ils appuyer ces développements? En auront-ils les moyens? En sentent-ils le besoin? On verra qu'au chapitre de la conscience des besoins, la situation en 2007 est peut-être un peu moins défavorable qu'en 2001.

Ce changement de paradigme est en train d'arriver au niveau de l'école publique. Après avoir aboli toute trace de confessionnalité dans les écoles québécoises (ce qui exigea une révision de la Constitution canadienne), le ministère de l'Éducation planifie la mise en place à l'automne 2008 d'un programme d'éthique et de culture religieuse offert à tous les niveaux du primaire et du secondaire ${ }^{19}$. Les nouvelles habitudes de connaissance vont être transmises aux enfants et adolescents ${ }^{20}$. L'implantation expérimentale dans quelques établissements choisis navigue avec succès entre deux récifs qui sont prêts à causer le naufrage de cette nouvelle barque. D'une part, on entend les critiques de ceux qui craignent un regain des superstitions et voudraient que ces heures de classe servent plutôt à accroître l'enseignement des mathématiques. D'autre part, il y a tous ceux qui appréhendent que le christianisme n'aura pas le nombre d'heures ni l'approche qu'il mérite. On voit vite que c'est la notion même de culture appliquée à la religion qui reste en travers de la gorge. Des deux côtés, les adversaires rejettent la notion de pluralisme des cultures et le besoin d'une certaine familiarité avec les concitoyens qui sont attachés à d'autres cultures religieuses.

$$
+++
$$

19. Voir MINISTERE DE L'ÉdUCATION, DU LOISIR ET DU SPORT, «La mise en place d'un programme d'éthique et de culcure religieusen, www.mels.gouv.qc.ca/lancement/Prog_ethique_cult_reli/ındex.asp (16 octobre 2007).

20. Voir Nancy BOUCHARD, Ethique et culture religieuse d̀ l'école, Québec, Presses de l'Unıversité du Québec, 2006 et Georges LEROUX, Ethique, culture religieuse, daalogue. Arguments pour un programme, Montreal, Fides, 2007. 
Pourtant, on enseigne la géographie. Les petits Québécois ne me semblent pas avoir été traumatisés par la découverte qu'il y a cinq continents habités. On leur a appris qu'il y a ''Australie, par exemple, et les Australiens. Très vite, ils savent reconnaître les kangourous, même s'ils n'en voient jamais dans leurs forêts. Avec le temps, ils seront même appelés à connaître le sens du mot marsupiaux. Il y a, je dirais, un certain plaisir esthétique à savoir que les kangourous existent, même si ce savoir n'est pas d'utilité immédiate. On en donne des peluches aux enfants. Cela élargit leur imagination. Mais cela menace-t-il leur identité? Leur chambre devrait-elle n'être peuplée que de castors? Le rappel de la dimension esthétique de la vie me semble crucial en matière d'éducation et d'initiation à la réalité des cultures. On montre l'art de la Renaissance italienne aux classes du secondaire; est-ce chercher à en faire des Italiens, ou seulement des Québécois éduqués? Informer les élèves des pratiques et croyances des hindous, est-ce tenter de les séduire et poser les bases d'une conversion? Où est-ce simplement les mettre au courant de l'existence de personnes d'une autre culture religieuse et les préparer à entrer avec aisance en relations civiles avec elles? Le gouvernement a gagé que l'interculturalité et la connaissance mutuelle au sein de la nation québécoise doivent inclure une certaine familiarité avec le domaine religieux et cela me semble tout à fait sage.

Les élections de mars 2007 ont bouleversé la composition de l'Assemblée nationale, et la question des religions a surgi brusquement et nettement dans les discours électoraux. Les articles recueillis dans le dossier de la revue Éthique publique publié au printemps 2007 et intitulé "L'aménagement de la diversité culturelle et religieuse " ${ }^{21}$ prennent note de ces événements récents. Une première partie donne des études comparatives dans une perspective de philosophie politique. J'en retiens le plaidoyer en faveur du recours à la notion de tolérabilité pour l'analyse des politiques publiques. La tolérabilité ne donne pas de normes, mais la notion de tolérance qui prétend le faire est trop vague pour aider à la gestion de la diversité.

La deuxième partie apporte des analyses et réflexions sur les disputes québécoises au sujet des "accommodements raisonnables". Les neuf auteurs abordent différents dossiers, mais reviennent sur les mêmes thèmes, en les éclairant de manières différentes. Je tenterai de regrouper les thèmes plutôt que d'examiner les articles un par un. Rappelons les faits saillants. Au

$$
+4
$$

21. Ethıque publique, vol. 9, $\mathrm{n}^{\circ} 1$, «'aménagement de la diversuté culturelle et religieusen, prıntemps 2007. 
cours des dernières années, des juifs, des musulmans et des sikhs de très stricte observance se rendirent fort visibles. Trois causes célèbres donnèrent lieu à des tollés médiatiques. Des juifs ultra-orthodoxes obtinrent le droit d'installer des erouvim, minces fils placés en hauteur au travers des rues, pour circonscrire un territoire à l'intérieur duquel les croyants peuvent marcher durant le sabbat. Des musulmanes se mirent à porter le voile; en général le foulard couvrant les cheveux, rarement le modèle qui recouvre tout le visage sauf les yeux. Et un écolier sikh obtint le droit de porter son kirpan (un poignard) en allant à l'école, dans un étui de bois puis de coton cousu. Ces causes furent judiciarisées en vertu de la Charte des droits et libertés; la première et la troisième furent tranchées par la Cour suprême du Canada. Dans son jugement sur le kirpan, la Cour a noté que beaucoup d'hommes sikhs ne portaient pas le kirpan et que d'autres en portaient un symbolique, petit et en bois ou en plastique. On peut donc être un sikh qui fréquente le temple et jouit de l'estime de sa communauté à Montréal sans porter le kirpan. Mais la Cour a déterminé, avec raison, que ce n'est pas à elle de juger des normes dans la religion des sikhs. Elle se borne à soutenir, au nom de la liberté de religion, le droit d'un individu à se situer dans l'absolu et à préférer désobéir à la loi scolaire plutôt que de ne pas porter son kirpan ${ }^{22}$. Il faut ajouter en passant que l'argumentation contre le droit au port du kirpan était faible et se bornait à avancer des raisons de sécurité (jouant sur des peurs attisées par le 11 septembre), alors que nul n'a pu donner un exemple où le kirpan avait été l'arme d'un crime. Un autre argument, qui a du poids à mes yeux, n'a pas été formulé. Depuis le $\mathrm{XV}^{e}$ siècle, l'Occident a réussi à démilitariser l'espace public. (Cela fut fait petit à petit et le processus n'est pas encore achevé aux États-Unis.) Confier la protection de sa personne à la loi est un trait de la société civile que je ne souhaite pas voir battu en brèche.

Ce qui a excité l'opinion avant tout, c'est la question de la place de la religion, en particulier de celle "des autres", dans l'espace public. Car certains ont l'impression que la religion catholique s'est faite discrète, mais que les autres se font envahissantes. Je laisse aux juristes le soin d'entrer dans leur technicité et le pourquoi du recours par les tribunaux à la notion d'accommodements raisonnables.

Une première observation d'ensemble s'impose sur les conséquences de la judiciarisation. "Accommodement raisonnable" désigne aussi

$$
+++
$$

22. Rappelons aux purs ex durs de la laïcité que la résistance aux dogmes des croyants ne se fir pas que par des laïes. L'histoire est remplie de mouvements religieux qui s'opposèrent jusqu'au martyre à une religion dominante. 
les processus quotidiens de bon voisinage, qui roulent en général sur le donnant donnant, mais sont parfois mis dans les mains des avocats puis des juges. Quand il y a judiciarisation au nom de la liberté de religion, l'une des parties sort gagnante, l'autre perdante. Le débat public sur les accommodements a semblé admettre que les droits conquis par les minorités religieuses étaient faits aux dépens de la majorité. On apprit que les administrations publiques faisaient beaucoup de concessions; le service des permis de conduire lors des examens ne mettait pas dans la voiture un inspecteur homme avec certaines candidates, ni une inspectrice avec certains candidats. Beaucoup ont conclu de ces histoires que ce qui était accordé aux uns était ôté aux autres ${ }^{23}$. Il en est résulté une réaction politique identitaire dont le poids s'est fait sentir aux urnes. Les conservateurs les plus encroûtés ont vite assimilé le discours des féministes pour renforcer leur islamophobie. Un sondage a aussi montré que des Québécois n'hésitaient pas à dire qu'ils étaient "un peu racistes" pour exprimer leur sentiment qu'il y avait des limites à la tolérabilité. Le moment vint où "le peuple" voulut dire aux autres que c'était à eux de s'adapter.

Une seconde observation est avancée par un analyste des expressions de frustration populaire. Il voit là une occasion de dépasser l'alternative entre nation civique et nation ethnique pour parler de communauté juridique et de communauté morale. Le droit existe et le système fonctionne pour dire le droit dans les nouvelles circonstances. Mais il y a aussi une communauté morale qui, dans l'ordinaire, prévient ou résout les disputes. Cette communauté ne peut pas exister entre groupes qui vivent isolés et ne se parlent pas. Les lois linguistiques visent à faciliter l'émergence d'une telle communauté, mais ne peuvent pas en garantir l'émergence. Pour cela, il faut un peu de proximité et qu'un minimum de connaissance réciproque circule entre les groupes. Signalons le minidébat qui a eu lieu au sujet des jeunes musulmanes exclues d'un tournoi de taekwondo parce qu'elles portaient un foulard sous leur casque réglementaire. L'arbitre a justifié sa décision par les statuts de la Fédération internationale. Puis, certains ont avancé l'argument sécuritaire. Ceux d'en face ont répliqué que cela ne tenait pas la route. Comme historien, je propose une lecture plus vaste des enjeux. Au début du $\mathrm{XX}^{\mathrm{e}}$ siècle, les combats de boxe en Amérique du Nord opposaient des champions à forte identité ethnique. C'était bon pour le business, remplissait

$$
4+
$$

23. Cela certes arriva. Un service social bien intentionné pria les maris de ne plus venır aux cours de préparation à l'accouchement. La raison écat qu'un mari (qu ne voulait pas venir) ne voulatt pas que sa femme $y$ alle s'il $y$ avair des hommes dans la salle. 
les arènes de foules enthousiastes - et hostiles l'une à l'autre. Il y avait des contestations de l'arbitre, des bagarres, des émeutes, des blessés et parfois des morts. On connait aujourd'hui les débordements qui accompagnent certains matches de football en Europe. L'uniforme des équipes sportives, strictement interprété, est donc à mes yeux le symbole de l'adhésion complète du joueur à un code sportif fort: fair-play, soumission prompte aux décisions de l'arbitre, confiance que la Fédération désigne des arbitres neutres, etc. Voici encore un acquis (fragile) de notre société civile qui ne devrait pas être ébranlé. Je ne prétends pas que c'est un argument décisif pour le litige au sujet des jeunes sportives du taekwondo, mais j'y vois une considération à verser dans le débat, même si elle est un peu compliquée pour certains médias.

Une troisième observation porte sur la distinction entre les libertés et les droits. Un inculpé qui ne comprend pas la langue de la Cour a droit à un traducteur, qui fait son travail aux frais de la Cour. La liberté de religion est une liberté que l'on exerce à ses propres frais. Une zone grise est créée par les politiques du multiculturalisme qui subventionnent certaines activités de minorités culturelles. Or, ces politiques sont remises en question. La résistance au multiculturalisme à la mode au Canada anglais devient plus ferme lorsqu'il s'agit de demandes de politiques publiques en faveur de la diversité religieuse. Ainsi, peu d'auteurs (ou de citoyens) accorderont que la liberté religieuse donne le droit à avoir un lieu de prière disponible sur les lieux de son emploi. (Ceci n'empêche pas un employeur ou un établissement d'en fournir un.) Mais les précédents établis au Québec ne vont pas du tout dans le sens de l'opinion qui veut minimiser la présence des convictions religieuses dans l'argumentaire public. Les croyants qui préferent une école "confessionnelle» à l'école publique ont pu convaincre l'État et édifier au fil des décennies un réseau d'établissements financés à $60 \%$ par les deniers publics. Ce sont surtout les catholiques et les juifs qui se sont prévalus de cette loi. Cela donne-t-il aux musulmans et aux sikhs le droit le plus strict d'en faire autant ${ }^{24}$ ?

Une quatrième observation porte sur le fait que le jeu se joue à plusieurs. Si la réaction identitaire oppose nous et les autres, le cas scolaire montre que le jeu est multipolaire. Beaucoup d'approches intellectuelles contemporaines traitent de l'altérité en général. (J’y vois là une conséquence

$$
++4
$$

24. Il convient aussi de noter que la Charte des droits et libertés exclut les organisations religieuses de son champ d'application. En 1974, il s'agissait d'empêcher des femmes de faire des procès à l'Église catholıque pour discrimination dans l'accès à la prêtrise. 
de vulgates psychanalytiques.) Dans la société, les adultes rencontrent des autres qui sont différents les uns des autres. Opposer les Québécois de souche aux immigrants récents remplace la complexité d'un pluralisme réel par un dualisme de pensée. À l'ère de l'autre défini a succédé aujourd'hui celle de l'autre indéfini ${ }^{25}$. Les immigrants aussi sont impliqués dans un possible jeu d'alliances changeantes. Les musulmans notent que le dimanche est jour férié et que les juifs ont obtenu des accommodements pour leur sabbat. Pourquoi alors les musulmans n'obtiendraient-ils pas des droits équivalents pour leur vendredi? La situation est ironique, car le vendredi musulman n'est pas un sabbat; la seule obligation est d'aller à la prière publique - qui dure une heure. Donc, cette revendication identitaire qui se veut traditionnelle est en fait une adaptation aux mœurs judéo-chrétiennes.

Que beaucoup grossissent le diable sur la muraille ne doit pas nous empêcher de voir la réalité politique créée par les fondamentalismes ou intégrismes religieux. Des idéologies politiques et religieuses, fort visibles chez quelques musulmans mais présentes ailleurs, prônent une société faite de nous sans les autres. L'ultramontain Louis Veuillot (1813-1883) est réputé avoir lancé à la tête des républicains la formule "Quand je suis le plus faible, je vous demande la liberté, parce que tel est votre principe; mais quand je suis le plus fort, je vous l'ôte, parce que tel est le mien ${ }^{26}$." J'ai lu une petite brochure (en anglais) incitant les musulmans à fonder des écoles confessionnelles, car la loi l'autorise, et ajoutant qu'une fois que les musulmans seront la majorité au Canada, la loi sera abolie et toutes les écoles seront islamiques. La contradiction est totale: on prend la parole pour s'adresser à d'autres citoyens dont on se prépare à nier la citoyenneté. Pourquoi prétendre faire société avec des personnes avec lesquelles on ne veut pas, au fond, faire société? Il n'y a pas de société sans réciprocité. Le fondamentalisme n'est pas simplement un extrémisme religieux; c'est aussi un péril moral et politique.

En société démocratique, ceux qui préferent ignorer tous les autres ou ne veulent les rencontrer que sur le mode de l'opposition opèrent une rupture qui n'est pas sans conséquence. (Le phénomène au Québec est renforcé par l'existence de ghettos linguistiques anglophones.) On sait que l'ignorance de la loi n'est pas une excuse. Devrait-on ajouter que l'ignorance de ses concitoyens ne saurait être une excuse? C'est certes beaucoup

$$
++
$$

25. À moins que l'on ne voie que les musulmans pour en faire un nouvel aurre défini comme dangereux, un choix qui seraut renforcé par une tendance planétaire.

26. Voir * Les Citations", www.lescicacions.net/citations/aureurs/Louis_Veuillor.html (14 janvier 2008). 
demander, mais j'hésiterai pour ma part à maintenir qu'il y a un droit fondamental de rester ignorant. Certes, beaucoup le font sans peine apparente, et nous avons tous indubitablement des taches obscures. Mais ces carences n'existent pas sans péril. Dans une communauté nationale, on ne fait pas que posséder des droits, on veut aussi être reconnu, on souhaite jouir de l'estime. Et la conversation dans le quotidien reste le meilleur moyen de gagner une bonne réputation. On a pu le voir à la télévision: quand des musulmanes à foulard s'expliquent en français en milieu pluraliste, elles ne manquent pas de gagner le respect - et parfois l'estime. Mais il n'y a pas que les vertus du dialogue. Il y a aussi celle de la connaissance des faits, des données objectives. De nombreux musulmans ont immigré au Québec parce qu'ils voulaient fuir la dérive islamiste dans leur patrie. La plupart des sikhs ne portent pas le kirpan. Et rares sont les juifs qui ont besoin de l'erouv pour vivre leur sabbat.

Le parcours à travers les cinq livres a commencé avec un épisode dans l'histoire du catholicisme québécois, pour nous amener à l'examen de problèmes d'éthique à l'intérieur du catholicisme puis dans la société en général. On a donc débouché sur l'étude de phénomènes sociaux. Par la suite, cela a débordé sur des problèmes de droit et un grand enjeu politique. Cette séquence des problèmes repose, je crois, sur une nécessité intrinsèque. Malgré ce que disent les militants du mouvement laïque sur l'apport de la science et la querelle entre raison et religion, je ne pense pas que la modernité soit caractérisée, avant tout, par l'essor et l'impact des sciences naturelles, quelque important que cela soit pour la philosophie de la connaissance. Je crois plutôt que la vraie vague de fond qui a bouleversé le monde, c'est la montée de "l'égalité comme principe fondamental de l'ordre social ${ }^{27}$ ". La religion autrefois légitimait l'inégalité (temporaire) et donnait à l'ordre des choses un parfum d'éternité. Mais, depuis 1800, l'Occident manque de légitimations pour l'inégalité entre les hommes. (Les néolibéraux, auxquels on signale que l'écart entre les riches et les pauvres s'accroît partout, en sont réduits à répéter que tous peuvent devenir riches et qu'à la longue ceux-ci deviendront plus nombreux.) L'argument de Tocqueville me semble imparable: depuis que la religion ne légitime plus l'inégalité entre les humains, ceux-ci sont précipités dans une existence faite de rivalités sociales et d'inquiétude individuelle. La "passion du bien-être" domine la vie des citoyens qui ont la chance de vivre en société démocratique. Autrefois, une

$$
+4
$$

27. Voir Olivier MICHAUD, *Tocqueville, inquiet de notre obsession maladive pour la santén, Le Devoir, 24 juın 2007 , p. B6. 
incorrigible inégalité de conditions forçait les individus à se préoccuper d'autres biens. Maintenant, les humains sont absorbés entièrement "dans la recherche des jouissances permises". Tocqueville espérait néanmoins que "la religion" élèverait nos regards vers des objectifs et des biens moins immédiats ${ }^{28}$.

Tocqueville semble se placer sur le terrain des apologétiques profanes qui assurent que toutes les sociétés ont besoin d'une religion. Dans son essai "Faut-il défendre la religion?", Jacques Bouveresse a bien montré que cette position (que l'on trouve chez Auguste Comte et Émile Durkheim) est beaucoup trop vague et peut être mise au service de n'importe quelle espèce de religion ${ }^{29}$. Ce n'est pas l'opinion de Tocqueville. La force de ses écrits vient du fait qu'il trouve une heureuse synergie entre la démocratie et la religion telles que pratiquées par les catholiques et les protestants aux ÉtatsUnis dans les années 1830. Il fait dépendre l'avenir de la démocratie d'une transformation de la religion. Il ne nous invite pas à céder à d'apparentes nécessités sociales ou politiques ${ }^{30}$, mais plutôt à inclure la réflexion critique sur la religion dans les tâches d'une philosophie politique ${ }^{31}$. C'est bien à l'art de vivre ensemble qu'il me semble urgent d'atteler toutes les forces de la raison.

Il n'en reste pas moins que la religion telle que nous la connaissons donne une lignée d'exemples d'individus qui se soustraient aux échanges sociaux quotidiens pour figer leur agir et leur monde sous les ordres de l'éternité. Notre législation le reconnaît. Ce n'est plus l'Église, mais la conscience qui est la "machine" pour s'opposer au temps. (Marcher au martyre en groupe n'est pas s'opposer au temps; cela reste du politique, car il y a ceux qui mènent et ceux qui sont menés, ceux qui survivent et ceux qui meurent.) Quand on réalise que le système quotidien d'échanges et de dons caractérise aussi une société comme la mafia (avec certes de fortes contraintes - mais quelle société est sans contrainte?), il faut bien admettre qu'une philosophie politique doit prévoir la résistance de l'individu, même sa

$$
4+
$$

28. On lit les ouvres principales de Tocqueville avec d'excellentes introductions dans Alexis de TOCQUEVILLE, De la démocratie en Amértque; Souventrs; Lancten régtme et la révolutron, introductions et notes de Jean-Claude Lamberti et de Françoise Mélonio, Paris, Laffont, 1986. Pour ses réflexions sur ce qu'il a vu au Canada français, voir Gérard BERGERON, Quand Tocqueville et Stegfred nous observaient. , Québec, Presses de l'Université du Québec, 1990.

29. On trouve cet essar dans Jacques BOUVERESSE, Peut-on ne pas crotre? Sur la véruté, la croyance et la foi, Marsellle, Agone, 2007, p. 39-250.

30. Bouveresse sıgnale que les penseurs qui n'ont pas de goût pour la teligion finissent souvent par s'accommoder de l'orthodoxie religieuse domınante pour raisons de politıque. La montée des incégrismes n'est pas à ses yeux le signe d'un "retour de la relıgion" mais plurốc le signe d'un recours à la relıgıon pour des fins non relıgıeuses, le plus souvent politiques (ibud, p. 62).

31. Vour Michel DESPLAND, Les hierarchies sont ébranlées. Polutrques et théologzes au Xex stècle, Montréal, Fides, 1998. 
résistance absolue et apparemment irrationnelle. Ce qui nous amène aux pages de Thoreau sur la désobéissance civile, "pour raison de conscience", et par là aux réalités de la conviction religieuse. 